\title{
SOX: Neutrino Oscillometry in Borexino
}

\author{
Mikko Meyer* \\ Institut für Experimentalphysik, Universität Hamburg, Luruper Chaussee 149, 22761 \\ D-Hamburg, Germany \\ E-mail: mikko.meyer@desy.de
}

\section{on behalf of the Borexino/SOX collaboration:}

M. Agostini, K. Altenmüller, S. Appel, G. Bellini, J. Benziger, N. Berton, D. Bick, G. Bonfini, D. Bravo, B. Caccianiga, F. Calaprice, A. Caminata, P. Cavalcante, A. Chepurnov, M. Cribier, D. D’Angelo, S. Davini, A. Derbin, L. di Noto, I. Drachnev, M. Durero, A. Empl, A. Etenko, S. Farinon, V. Fischer, K. Fomenko, D. Franco, F. Gabriele, J. Gaffiot, C. Galbiati, C. Ghiano, M. Giammarchi, M. Göger-Neff, A. Goretti, M. Gromov, C. Hagner, T. Houdy, E. Hungerford, Aldo Ianni, Andrea Ianni, N. Jonquères, M. Kaiser, V. Kobychev, D. Korablev, G. Korga, D. Kryn, T. Lachenmaier, T. Lasserre, M. Laubenstein, B. Lehnert, J. Link, E. Litvinovich, F. Lombardi, P. Lombardi, L. Ludhova, G. Lukyanchenko, I. Machulin, W. Maneschg, S. Marcocci, J. Maricic, G. Mention, E. Meroni, M. Meyer, L. Miramonti, M. Misiaszek, M. Montuschi, V. Muratova, R. Musenich, B. Neumair, L. Oberauer, M. Obolensky, F. Ortica, L. Pagani, M. Pallavicini, L. Papp, L. Perasso, A. Pocar, G. Ranucci, A. Razeto, A. Re, A. Romani, R. Roncin, N. Rossi, S. Schönert, L. Scola, D. Semenov, H. Simgen, M. Skorokhvatov, O. Smirnov, A. Sotnikov, S. Sukhotin, Y. Suvorov, R. Tartaglia, G. Testera, M. Toropova, E. Unzhakov, C. Veyssière, M. Vivier, R. B. Vogelaar, F. von Feilitzsch, H. Wang, J. Winter, M. Wojcik, M. Wurm, Z. Yokley, O. Zaimidoroga, S. Zavatarelli, K. Zuber, G. Zuzel

\begin{abstract}
Several observed anomalies in the neutrino sector could be explained by a fourth (sterile) neutrino with a squared mass difference in the order of $1 \mathrm{eV}^{2}$ to the other three standard neutrinos. This hypothesis can be tested with an artificial kCi antineutrino (Ce-144/Pr-144) source deployed near or inside a large low background detector like Borexino. The SOX project (short baseline neutrino oscillation with Borexino) aims for the detection of sterile neutrinos and offers the almost unique possibility to observe the characteristic antineutrino oscillation pattern within the detector. The concept is summarized whereby particular focus is given to the antineutrino spectrum calculation.
\end{abstract}

XVI International Workshop on Neutrino Telescopes,

2-6 March 2015

Palazzo Franchetti - Istituto Veneto, Venice, Italy

\footnotetext{
* Speaker.
} 


\section{Introduction}

Several short-baseline neutrino experiments have reported anomalies which are inconsistent with the three active neutrino picture (LSND, MiniBooNE, GALLEX, SAGE) [1]. This might be explained by the existence of a fourth non-weakly interacting sterile neutrino ( $3+1$ model) with a relatively large squared mass difference of $\Delta m_{41}^{2}>0.1 \mathrm{eV}$.

The SOX project aims for the detection of sterile neutrinos and offers the almost unique possibility to observe the characteristic antineutrino oscillation pattern within the detector.

The upcoming analysis relies on a precise knowledge of the antineutrino spectral shape. This will be achieved by an experimental effort as well as theoretical calculations as outlined in the following.

\section{The SOX Experiment}

One powerful method to probe the neutrino anomalies is to place an intense $v_{e}$ or $\bar{v}_{e}$ source near or inside a large low background detector like Borexino $[2,3]$.

Borexino is a $300 \mathrm{t}$ spherical liquid scintillator detector located at the LNGS in Italy [4]. The main purpose of the experiment is the real-time detection of solar neutrinos [5]. As part of an $\mathrm{R} \& \mathrm{D}$ effort, Borexino has reached an unprecedented radio-purity and the concentration of ${ }^{238} \mathrm{U}$ and ${ }^{232} \mathrm{Th}$ is now as low as $10^{-19} \mathrm{~g} / \mathrm{g}$ [2]. The vertex resolution is about $15 \mathrm{~cm}$ at $1 \mathrm{MeV}$ and the energy resolution is $6 \% / \sqrt{E}$ [2] which makes Borexino an ideal candidate to search for sterile neutrinos in the mostly unexplored low $L / E$ region.

During the first phase of the experimental setup, an antineutrino source will be placed underneath the detector $\left(8.25 \mathrm{~m}\right.$ from the detector center). The detection of the $\bar{v}_{e}$ is then performed by the inverse beta decay (IBD, $p+\bar{v}_{e} \rightarrow e^{+}+n$ ) which was already successfully applied for the geo-neutrino analysis. The isotope pair ${ }^{144} \mathrm{Ce}-{ }^{144} \mathrm{Pr}$ has been identified as a suitable antineutrino emitter making the technical challenges in the production feasible. This isotope pair provides also a long half life to allow the production and transportation as well as a high Q value of $2.995 \mathrm{MeV}$ which is well above the IBD threshold. The activity of the source will be in the order of $100 \mathrm{kCi}$.

\subsection{Theoretical $\bar{v}_{e}$ Spectrum Modeling}

For the prediction of the IBD interactions inside the detector, the source activity has to be known with high precision in the order of $1 \%$. The activity measurement is performed by a calorimeter and depends strongly on the mean released energy which is directly linked to the $\bar{v}_{e}$ spectrum shape. Any uncertainty of the $\bar{v}_{e}$ spectrum shape will therefore affect the rate+shape sensitivity of the experiment.

The theoretical modeling of the $\bar{v}_{e}$ spectrum is based on Fermi's theory of $\beta$ decay:

$$
N_{\beta}(W)=K S_{n} W p\left(W-W_{0}\right)^{2} F(Z, W),
$$

where $S_{n}$ is the shape factor, $K$ is a normalization constant, $Z$ is the atomic number, $W=E_{e} / m_{e}+1$ is the total energy of the $\beta$ particle in units of electron mass, $p=\sqrt{W^{2}-1}$ is the momentum of the $\beta$ particle, and $W_{0}=Q / m_{e} \pm 1$ is the maximum $\beta^{\mp}$ particle energy. $E_{e}$ is the kinetic energy of the 


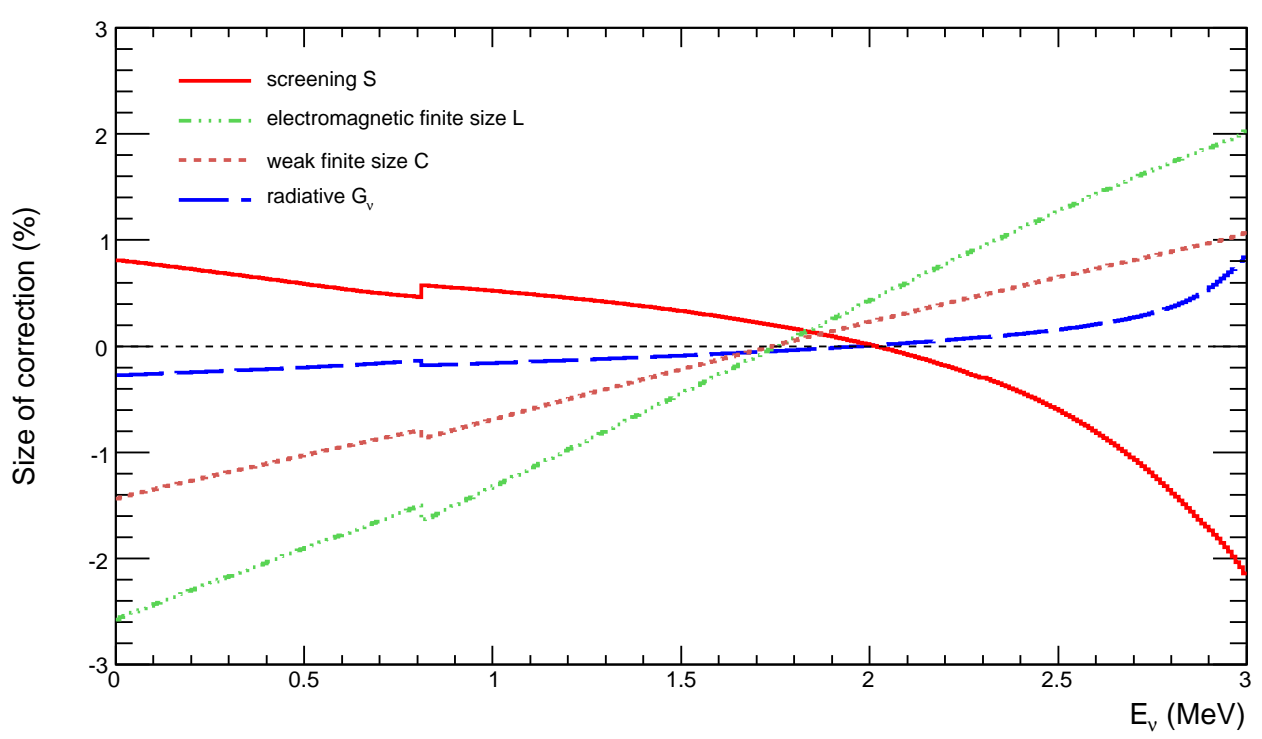

Figure 1: Relative size of various antineutrino spectrum corrections for the ${ }^{144} \mathrm{Pr}$ source. $E_{V}$ is the antineutrino energy.

$\beta$ particle and $Q$ is the end-point of the $\beta$ spectrum. The Fermi function $F(Z, W)$ is the leading QED correction to the $\beta$ spectrum shape and is given by

$$
F(Z, W)=2(\gamma+1)(2 p R)^{2(\gamma-1)} e^{\pi \alpha Z W / p} \frac{|\Gamma(\gamma+\imath \alpha Z W / p)|^{2}}{\Gamma(2 \gamma+1)^{2}} .
$$

The nuclear radius $R$ can be determined with the Elton formula. The $\bar{v}_{e}$ and $\beta$ spectra is in principle symmetric under energy conservation $W_{v}=W_{0}-W$ with the exception of different radiative correction for electrons and antineutrinos.

In addition, several sub-leading correction particularly such as finite size, screening of the atomic electrons and radiative effects have to be considered (see Figure 1). The size of each of these corrections can be in the order of a few percent as seen in Figure 1. The $\bar{v}_{e}$ spectrum modeling is based on the summary as outlined in [6]. Weak magnetism is expected to be negligible as the dominant ${ }^{144} \operatorname{Pr} \beta$ transition is a $0^{-} \rightarrow 0^{+}$non-unique first forbidden transition with no angular momentum change [7]. The dominant uncertainty arises then from the screening correction and from shape factors $S_{n}$ of the first non-unique forbidden transitions of ${ }^{144} \mathrm{Pr}$. As the shape factors for first non-unique forbidden transitions depend on matrix element further experimental studies are necessary to constrain the error for the rate+shape analysis. The other corrections listed above are generally well known and will affect the IBD interaction rate in the order of $1 \%$.

\subsection{Neutrino Oscillometry}

The unambiguous way to search for sterile neutrinos is the determination of the oscillation pattern in the distant dependent flux of a pure neutrino flavor over the entire oscillation length [8]. Assuming $\Delta m_{41}^{2} \sim 1 \mathrm{eV}^{2}$ as favored by global fits and an antineutrino energy of about $1 \mathrm{MeV}$, the 


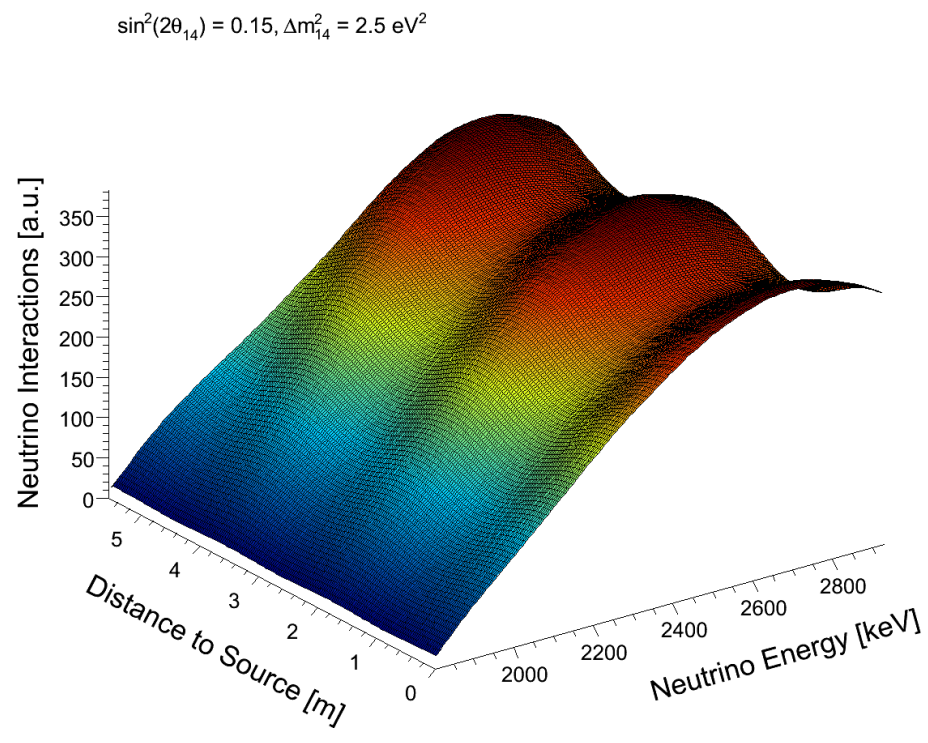

Figure 2: Oscillation pattern for an antineutrino source positioned in the center of the detector with an enhanced fiducial volume radius $(5.5 \mathrm{~m}$ instead of $4.25 \mathrm{~m})$. The neutrino energy is the product of the $\bar{v}_{e}$ spectrum and the IBD cross-section.

oscillations length will be in the order of a few centimeters to meters [8]. This makes Borexino an ideal candidate to resolve the oscillation pattern since the detector is big enough and offers a sufficient energy and vertex resolution (see Figure 2). This concept is known as neutrino oscillometry and is especially attractive in case the source is placed inside the detector. The high geometrical acceptance and interaction rate could than provide a definite proof or disproof of the long-standing neutrino anomalies (see Figure 2).

\section{Conclusion}

The ${ }^{144} \mathrm{Ce}-{ }^{144} \mathrm{Pr}$ antineutrino source will be placed in a tunnel underneath the large low background detector Borexino at the end of 2016. The calculation of the $\bar{v}_{e}$ spectrum shape is one of the key elements for the upcoming rate+shape analysis.

\section{References}

[1] C. Giunti, M. Laveder, Y. F. Li, Q. Y. Liu and H. W. Long, Phys. Rev. D 86, 113014 (2012)

[2] M. Pallavicini [Borexino Collaboration], PoS Neutel 2013, 026 (2013).

[3] G. Bellini et al. [Borexino Collaboration], JHEP 1308, 038 (2013)

[4] G. Alimonti et al. [Borexino Collaboration], Nucl. Instrum. Meth. A600, 568 (2009),

[5] G. Bellini et al. [Borexino Collaboration], Nature 512, no. 7515, 383 (2014).

[6] P. Huber, Phys. Rev. C 84, 024617 (2011) [Phys. Rev. C 85, 029901 (2012)]

[7] A. C. Hayes et al., Phys. Rev. Lett. 112, 202501 (2014)

[8] A. Gando et al., arXiv:1312.0896 [physics.ins-det]. 